\title{
Cerebral Microdialysis: A Metabolic Sensor Inside the Closed Box
}

\section{Samy Raheem Abdel*}

Department of Anaesthesiology and ICU, Asyut University Hospitals, Egypt

\section{Editorial}

The cranial vault was regarded by Monro-Kellie doctrine as a closed box containing the three components namely: brain, blood and cerebrospinal fluid. The brain is orders of magnitude, more complex than the heart and other vital organs yet its function has been more difficult to monitor being contained in a rigid closed box. Following an acute neurological catastrophe such as traumatic brain injury (TBI) or stroke, damage to the brain can occur at the time of the initial event. This damage is termed "primary brain injury". Regardless of the interventions performed to limit this primary injury, much of the primary damage may be irreversible. However, this primary brain injury initi $\neg$ ates a cascade of biochemical events that are potentially reversible. This process, termed "secondary brain injury", includes structural and chemical derangement as well as alterations in cerebral blood flow and oxygen delivery. Management of secondary brain injury is of fundamental importance to the treatment of patients with TBI, ischemic and hemorrhagic stroke, and global cerebral ischemia [1].

In neuroscience critical care unit (NICU), new technologies provide practitioners with vital information on intracranial dynamics in order to normalize pressure and optimize blood and oxygen delivery, hence improving the outcomes. Current strategies for management of essentially all acute brain disorders encountered in NICU are centered on the goal of limiting secondary brain injury. However, effective management of secondary brain injury relies on the ability to detect its occurrence, monitor its progress, and observe the response to the interventions, which often have their own risks. Monitoring of systemic parameters, such as blood-pressure level and peripheral oxygen saturation, with the hope of adequately protecting the brain from secondary injury proved to be insufficient. Therefore, current and future approaches in NICU emphasize the ability to directly monitor the brain and to develop better tools to facilitate the analysis and integration of these advanced data for real-time clinical care.

In 1966, Bito et al. [2] inserted membrane-lined sacks containing $6 \%$ dextran into the cerebral hemispheres of dogs. The sacks were removed 10 weeks later and the fluid they contained was analyzed for levels of amino acids. During the 1970s this technique was refined and developed into cerebral microdialysis (CMD), in which a perfusate solution is passed along a semi-permeable membrane inserted in the brain parenchyma, allowing continuous sampling of the brain extracellular fluid [3]. The only commercially available bedside CMD analyzer (CMA 600, Sweden) received approval of the Food and Drug Administration in the United States in 2002.

Cerebral microdialysis (CMD) involves placement of a small catheter into the brain parenchyma, either during surgery or through a burr hole, secured by a cranial bolt [4]. Extracellular concentrations of ischemic metabolites, such as lactate and pyruvate, can then be measured. The lactate: pyruvate ratio is currently the best marker of the brain redox state and an early biomarker of secondary ischemic injury. Glycerol, a marker of cell membrane damage, and glutamate, an excitatory amino acid, provide additional evidence of developing brain injury [5]. CMD has been used, in the NICU arsenal of multimodal monitoring, to monitor patients with TBI and sub-arachnoid hemorrhage $(\mathrm{SAH})$. It has also been used for monitoring patients during neurosurgical procedures, to evaluate the impact of therapeutic interventions and for profiling of new neuroprotective drugs [6].

CMD has been shown repeatedly to correlate with other components of multimodal brain monitoring, such as jugular venous saturation and brain tissue oxygenation [7]. Specifically, intracranial pressure and cerebral perfusion pressure have both been reported to correlate with CMD values [8], and manipulation of these parameters is often firstline bedside response to pathological CMD values. In addition, there is a large body of literature suggesting that CMD monitoring is able to predict poor outcome after TBI and SAH. There is also some evidence that CMD may assist clinical decision-making, such as management of cerebral perfusion pressure [8], guidance of hyperventilation [9] and the appropriateness of extensive surgical procedures [10].

A limitation to microdialysis is the slow transport of fluid through the catheter, therefore measured values rep $\neg$ resent cerebral events that occurred 2060 min earlier, depending on the collection interval. The use of new, larger-pore membranes provides the possibility of measuring the broader neuroinflammatory cascades [11]. Another limitation is that CMD reflects only local tissue biochemistry and the accurate placement of the catheter is, therefore, crucial. Furthermore, because there are wide variations in measured variables, trend data are more important than absolute values [6]. Recently, Nelson et al. [12] reported that the multitude of highly perturbed local chemistry seen with CMD in patients with TBI predominately represents long-term metabolic patterns over a period of days.

At present, microdialysis is generally considered only a research tool, despite being increasingly used to guide management [13]. A consensus document recognizing the increased use of microdialysis [5] has appeared with the aim of defining various issues in CMD use, such as catheter location and unreliable values of biomarkers. Although clinical experience is rapidly increasing, carefully designed prospective studies are required to determine the value of CMD as a cerebral monitor. However, because of its unique ability to contribute important information about the process of secondary brain injury, CMD has the potential to become established as a key component of multi-modality cerebral monitoring in NICU.

\section{References}

1. Fahy BG, Sivaraman V (2002) Current concepts in neurocritical care Anesthesiol Clin North America 20: 441-462.

*Corresponding author: M. Samy Raheem Abdel, Department of Anaesthesiology and ICU, Asyut University Hospitals, Egypt, E-mail: msraheem@doctors.org.uk

Received March 20, 2012; Accepted March 20, 2012; Published March 22, 2012

Citation: Raheem Abdel MS (2012) Cerebral Microdialysis: A Metabolic Sensor Inside the Closed Box. J Biosens Bioelectron 3:e106. doi:10.4172/21556210.1000e106

Copyright: (c) 2012 Raheem Abdel MS. This is an open-access article distributed under the terms of the Creative Commons Attribution License, which permits unrestricted use, distribution, and reproduction in any medium, provided the original author and source are credited. 
Citation: Raheem Abdel MS (2012) Cerebral Microdialysis: A Metabolic Sensor Inside the Closed Box. J Biosens Bioelectron 3:e106. doi:10.4172/21556210.1000 e106

2. Bito L, Davson H, Levin E, Murray M, Snider N (1966) The concentration of free amino acids and other electrolytes in cerebrospinal fluid, in vivo dialysate of brain, blood plasma of the dog. J Neurochem 13: 1057-1067.

3. Delgado JM, DeFeudis FV, Roth RH, Ryugo DK, Mitruka BM (1972) Dialytrode for long term intracerebral perfusion in awake monkeys. Arch Int Pharmacodyn Ther 198: 9-21.

4. Goodman JC, Robertson CS (2009) Microdialysis: is it ready for prime time? Curr Opin Crit Care 15: 110-117.

5. Bellander BM, Cantais E, Enblad P, Hutchinson P, Nordstrom CH, et al. (2004) Consensus meeting on microdialysis in neurointensive care. Intensive Care Med 30: 2166-2169.

6. Tisdall MM, Smith M (2006) Cerebral microdialysis: research technique or clinical tool. Br J Anaesth 97: 18-25.

7. Johnston AJ, Gupta AK (2002) Advanced monitoring in the neurology intensive care unit: microdialysis. Curr Opin Crit Care 8: 121-127.

8. Nordstrom C, Reinstrup P, Xu W, Gardenfors A, Ungerstedt U (2003) Assessment of lower limit for cerebral perfusion pressure in severe head injuries by bedside monitoring of regional energy metabolism. Anesthesiology 98: $809-814$

9. Marion DW, Puccio A, Wisniewski SR, Kochanek P, Dixon CE, et al. (2002) Effect of hyperventilation on extracellular concentrations of glutamate, lactate, pyruvate, and local cerebral blood flow in patients with severe traumatic brain injury. Crit Care Med 30: 2619-2625

10. Boret H, Fesselet J, Meaudre E, Gaillard PE, Cantais E (2006) Cerebra microdialysis and $\mathrm{P}(\mathrm{ti}) \mathrm{O} 2$ for neuro-monitoring before decompressive craniectomy. Acta Anaesthesiol Scand 50: 252-254.

11. Hutchinson PJ, O'Connell MT, Rothwell NJ, Hopkins SJ, Nortje J, et al. (2007) Inflammation in human brain injury: intracerebral concentrations of IL 1alpha, IL 1 beta, and their endogenous inhibitor IL 1ra. J Neurotrauma 24: 1545-1557.

12. Nelson DW, Thornquist B, MacCallum RM, Nyström H, Holst A, et al. (2011) Analyses of cerebral microdialysis in patients with traumatic brain injury: relations to intracranial pressure, cerebral perfusion pressure and catheter placement. BMC Med 9: 21.

13. Andrews PJ, Citerio G, Longhi L, Polderman K, Sahuquillo J, et al. (2008) NICEM consensus on neurological monitoring in acute neurological disease. Intensive Care Med 34: 1362-1370. 